\title{
Quasiparton distribution functions: Two-dimensional scalar and spinor QCD
}

\author{
Xiangdong $\mathrm{Ji}^{1,2, *}$ Yizhuang Liu, ${ }^{2,3, \dagger}$ and Ismail Zahed ${ }^{3,+}$ \\ ${ }^{1}$ Department of Physics and Astronomy, University of Maryland, College Park, Maryland 20742, USA \\ ${ }^{2}$ Tsung-Dao Lee Institute, Shanghai Jiao University, Shanghai, 200240, China \\ ${ }^{3}$ Department of Physics and Astronomy, Stony Brook University, Stony Brook, New York 11794-3800, USA
}

(Received 6 November 2018; published 15 March 2019)

\begin{abstract}
We construct the quasiparton distributions of mesons for two-dimensional QCD with either scalar or spinor quarks using the $1 / N_{c}$ expansion. We show that in the infinite momentum limit, the parton distribution function is recovered in both leading and subleading order in $1 / N_{c}$.
\end{abstract}

DOI: 10.1103/PhysRevD.99.054008

\section{INTRODUCTION}

Light-cone distribution amplitudes are central to the description of hard exclusive processes with large momentum transfer. They account for the nonperturbative quark and gluon content of a hadron in the infinite momentum frame. Using factorization, hard cross sections can be split into soft partonic distributions convoluted with perturbativly calculable processes. The partonic distributions are inherently nonperturbative. They are currently estimated using experiments, lattice simulations, or models.

Recently, one of us [1] has suggested that the light-cone hadronic wave functions can be recovered from Euclidean correlators in hadronic states using instead quasiparton distribution functions through pertinent renormalization in the infinite momentum limit. Preliminary lattice simulations have proven very promising $[2,3]$. The purpose of this letter is to explore this construct in two-dimensional scalar and spinor QCD in the nonperturbative $1 / N_{c}$ expansion.

Two-dimensional scalar QCD has a smooth large $N_{c}$ limit with a confining spectrum [4-6]. In this model, the current correlators exhibits many features of fourdimensional QCD in contrast to two-dimensional spinor QCD [7]. In the deep inelastic regime, the results exhibit expected scaling laws, and are overall in support of the Feynman partonic picture and the light-cone expansion. In this paper, these two models will be used interchangeably to test the concept of the quasidistributions in a

\footnotetext{
xji@physics.umd.edu

yizhuang.liu@sju.edu.cn

*ismail.zahed@stonybrook.edu
}

Published by the American Physical Society under the terms of the Creative Commons Attribution 4.0 International license. Further distribution of this work must maintain attribution to the author(s) and the published article's title, journal citation, and DOI. Funded by SCOAP . nonperturbative context, as they differ by a minor change in the algebra of the pertinent bosonic operators. Specifically, we construct the quasiparton distributions for both scalar and spinor QCD in leading and subleading order in $1 / N_{c}$ and show that they merge with the expected light-cone distributions in the infinite momentum limit without additional renormalization. Our leading conclusion for two-dimensional spinor QCD is in agreement with a recent study [8].

This paper consists of several new results: (i) a bosonization of two-dimensional scalar and spinor QCD in the axial gauge, based on a closed form algebra valid to all orders in $1 / N_{c}$; (ii) a derivation of the parton quasidistribution function for two-dimensional scalar QCD in leading order in $1 / N_{c}$ with both leading and subleading order in $1 / P^{2}$; (iii) a smooth reduction of the parton quasidistribution function to the distribution function in the infinite momentum frame except at $x=0,1$; (iv) a derivation of the parton distribution and quasidistribution functions in spinor QCD at subleading order in $1 / N_{c}$.

The organization of the paper is as follows: in Sec. II, we discuss a canonical quantization of two-dimensional scalar QCD in the axial gauge. We make explicit the Hamiltonian of the model in leading order in $1 / N_{c}$ using bosonized fields. Some renormalization issues are also discussed. In Sec. III, we make explicit the wave function for scalar QCD in the light-cone limit. In Sec. IV, we construct the quasiparton distribution function in leading order in $1 / N_{c}$, and show that it reduces to the light-cone wave function in the infinite momentum limit. We also discuss the leading correction in $1 / P$. In Sec. V, we show how to generalize the bosonization scheme algebraically for both scalar and spinor $\mathrm{QCD}$, and use it for a systematic organization of the operators in $1 / N_{c}$. This scheme is used in Secs. VI and VII to correct the light-cone parton distribution and quasidistribution in spinor twodimensional QCD through standard perturbation theory. 
We show that the subleading corrections to the quasiparton distribution function merges with the parton distribution function in the infinite momentum limit without renormalization. Our conclusions are in Sec. VIII. In the Appendix, we summarized some elements of twodimensional spinor QCD pertinent for our canonical analysis both in light-cone and axial gauge.

\section{QUANTIZATION OF SCALAR QCD IN AXIAL GAUGE}

We first discuss the general structure of the Hamiltonian in two dimensions for scalar $\mathrm{SU}\left(\mathrm{N}_{c}\right)$ QCD in the axial gauge $A_{1}=0$. The same discussion for two-dimensional spinor QCD in both the light-cone and axial gauge is summarized briefly in the Appendix. The starting Lagrangian is

$$
\mathcal{L}=\frac{1}{2} \operatorname{Tr} F_{01}^{2}+\left(D^{\mu} \phi\right)^{\dagger} D_{\mu} \phi-m^{2} \phi^{\dagger} \phi,
$$

where $\phi_{\alpha}(x)$ with $\alpha=1, \ldots, N_{c}$ is a charged scalar field in the fundamental representation of $\mathrm{SU}\left(\mathrm{N}_{c}\right)$. In terms of the canonical momenta $\pi^{\dagger}=\Pi_{\phi}=\left(D_{0} \phi\right)^{\dagger}$ and $\pi=\Pi_{\phi^{\dagger}}=D_{0} \phi$, the corresponding Hamiltonian reads

$$
\begin{aligned}
H= & \int d x\left(\pi^{\dagger} \pi+\left|\partial_{1} \phi\right|^{2}+m^{2}|\phi|^{2}\right. \\
& \left.+i g \operatorname{Tr} A_{0}\left(\pi \phi^{\dagger}-\phi \pi^{\dagger}\right)-\frac{1}{2} \operatorname{Tr}\left(\partial_{1} A_{0}\right)^{2}\right) .
\end{aligned}
$$

The equation of motion for $A_{0}$ is a constraint equation that can be solved in terms of $\phi, \pi$ to yield the canonical Hamiltonian

$$
\begin{aligned}
H= & H_{0}+H_{\mathrm{int}} \\
= & \int d x\left(\pi^{\dagger} \pi+\left|\partial_{1} \phi\right|^{2}+m^{2}|\phi|^{2}\right) \\
& +\frac{g^{2}}{2} \int d x\left(J^{a} \frac{-1}{\partial_{1}^{2}} J^{a}\right),
\end{aligned}
$$

with the current $J^{a}=i\left(\phi^{\dagger} T^{a} \pi-\pi^{\dagger} T^{a} \phi\right)$. To proceed further, we will use a freelike representation for the charged field and its conjugate

$$
\begin{aligned}
\phi_{\alpha} & =\int \frac{d k}{\sqrt{4 \pi E_{k}}} e^{-i k x}\left(a_{k}+b_{-k}^{\dagger}\right)_{\alpha} \\
\left(\pi^{\dagger}\right)_{\alpha} & =i \int \frac{d k}{\sqrt{4 \pi E_{k}}} e^{i k x} E_{k}\left(a_{k}^{\dagger}-b_{-k}\right)_{\alpha},
\end{aligned}
$$

with $\alpha=1, \ldots, N_{c}$ the color index. Instead of the free dispersion law $E_{k}=\sqrt{k^{2}+m^{2}}$, we will use an arbitary $E(k)$ that will be fixed self-consistently below in the large $N_{c}$ limit (planar approximation), with $E_{k} \rightarrow|k|$ asymptotically.

\section{A. Hamiltonian to order $1 / \sqrt{N_{c}}$}

The Hamiltonian (3) is up to quartic in $a_{k}, a_{k}^{\dagger}, b_{k}, b_{k}^{\dagger}$. To analyze it we use the bosonization method as developed in the context of nonrelativistic many-body systems [9], and adapted to relativistic QCD in $1+1$ dimensions [10] to which we refer for more details. More specifically, we define the bilocal and color-singlet operators

$$
\begin{aligned}
M\left(k_{1}, k_{2}\right) & =\frac{1}{\sqrt{N_{c}}} \sum_{\alpha} a_{\alpha}\left(k_{1}\right) b_{\alpha}\left(k_{2}\right) \\
N\left(k_{1}, k_{2}\right) & =\sum_{\alpha} a_{\alpha}^{\dagger}\left(k_{1}\right) a_{\alpha}\left(k_{2}\right) \\
\bar{N}\left(k_{1}, k_{2}\right) & =\sum_{\alpha} b_{\alpha}^{\dagger}\left(k_{1}\right) b_{\alpha}\left(k_{2}\right),
\end{aligned}
$$

which are readily shown to form a closed algebra,

$$
\begin{aligned}
{\left[M_{12}, M_{34}^{\dagger}\right] } & =\delta_{13} \delta_{24}+\frac{s}{N_{c}}\left(\delta_{13} \bar{N}_{42}+\delta_{42} N_{31}\right) \\
{\left[M_{12}, N_{34}\right] } & =\delta_{13} M_{42} \quad\left[M_{12}, \bar{N}_{34}\right]=\delta_{23} M_{14} \\
{\left[M_{12}, M_{34}\right] } & =\left[N_{12}, \bar{N}_{34}\right]=0 \\
{\left[N_{12}, N_{34}\right] } & =\delta_{23} N_{14}-\delta_{14} N_{32},
\end{aligned}
$$

with $N_{12}^{\dagger}=N_{21}$, and the short hand notation $M_{12} \equiv$ $M\left(k_{1}, k_{2}\right)$ and so on. The sign assignment for the bosonization of scalar QCD is $s=+1$ as all underlying operators are bosonic. It is $s=-1$ for the bosonization of spinor QCD. $M^{\dagger}$ creates a mesonlike state composed of a pair of a charged scalar quark and its conjugate in the color-singlet representation, while $M$ annihilates the corresponding pair. Using (5) and the identity

$$
\sum_{a}\left(T^{a}\right)_{i j}\left(T^{a}\right)_{k l}=\delta_{i l} \delta_{k j}-\frac{1}{N_{c}} \delta_{i j} \delta_{k l},
$$

the Hamiltonian (3) now reads to order $1 / \sqrt{N_{c}}$ as

$$
\begin{aligned}
H \approx & H_{2}+H_{4} \\
H_{2}= & \int d k \Pi^{+}(k)(N(k)+\bar{N}(k)) \\
& +\sqrt{N_{c}} \int d k \Pi^{-}(k)\left(M(k)+M^{\dagger}(k)\right) \\
H_{4}= & \frac{\lambda}{16 \pi} \int d k_{1} d k_{2} d k_{3} d k_{4} \frac{\delta\left(k_{1}+k_{2}+k_{3}+k_{4}\right)}{\left(k_{1}+k_{2}\right)^{2}} \\
& \times\left(-2 f_{+}\left(k_{1}, k_{2}\right) f_{+}\left(k_{3}, k_{4}\right) M^{\dagger}\left(k_{1}, k_{4}\right) M\left(-k_{2},-k_{3}\right)\right. \\
& +f_{-}\left(k_{1}, k_{2}\right) f_{-}\left(k_{3}, k_{4}\right) M^{\dagger}\left(k_{1}, k_{4}\right) M^{\dagger}\left(k_{3}, k_{2}\right) \\
& \left.+f_{-}\left(k_{1}, k_{2}\right) f_{-}\left(k_{3}, k_{4}\right) M\left(k_{1}, k_{4}\right) M\left(k_{3}, k_{2}\right)\right) .
\end{aligned}
$$


Here, $\lambda=g^{2} N_{c}$ is the standard $\mathfrak{t}^{\prime}$ Hooft coupling. We have made use of the notation $M(k) \equiv M(k,-k)$, $N(k) \equiv N(k, k)$, and defined

$$
\begin{aligned}
\Pi^{ \pm}(k) & =\frac{1}{2}\left(\frac{k^{2}+m^{2}}{E_{k}} \pm E_{k}\right)+\lambda \int \frac{d k_{1}}{8 \pi} \frac{\frac{E_{k_{1}}}{E_{k}} \pm \frac{E_{k}}{E_{k_{1}}}}{\left(k+k_{1}\right)^{2}} \\
f_{ \pm}\left(k_{1}, k_{2}\right) & =\sqrt{\frac{E_{2}}{E_{1}}} \pm \sqrt{\frac{E_{1}}{E_{2}}} .
\end{aligned}
$$

For a consistent expansion in $1 / N_{c}$, we can eliminate the $\sqrt{N_{c}}$ term in (8) by setting $\Pi^{-}(k)=0$. The result is an integral equation for $E(k)$

$$
\frac{k^{2}+m^{2}}{E_{k}}-E_{k}+\frac{\lambda}{4 \pi} \int d k_{1}\left(\frac{E_{k_{1}}}{E_{k}}-\frac{E_{k}}{E_{k_{1}}}\right) \frac{1}{\left|k+k_{1}\right|^{2}}=0 .
$$

We now follow [10] and note that the bilocal and color singlet operators $N, N^{\dagger}$ can be recast in terms of the bilocal mesonic and color singlet operators $M, M^{\dagger}$

$$
\begin{aligned}
& N(k, p)=\int d q M^{\dagger}(k, q) M(p, q) \\
& \bar{N}(k, p)=\int d q M^{\dagger}(q, k) M(q, p),
\end{aligned}
$$

without affecting the commutation rules (6) in leading order in $1 / N_{c}$. This replacement is justified in the confined sector. Confinement implies that the creation of a charged scalar through $a_{\alpha}^{\dagger}\left(k_{1}\right)$ has to be always stringed to a meson creation, say $M^{\dagger}\left(k_{1}, q\right)$, and its annihilation through $a_{\alpha}\left(k_{2}\right)$ stringed to a meson annihilation, say $M\left(q, k_{2}\right)$. In nonrelativistic many-body physics, the representation (11) is known as the Holstein-Primakoff representation [9]. Inserting (11) into (8) and using the gap equation (9) yields the leading Hamiltonian to order $1 / \sqrt{N_{c}}$

$$
\begin{aligned}
H \approx & \int d p d q\left(\Pi^{+}(p)+\Pi^{+}(q)\right) M^{\dagger}(p, q) M(p, q) \\
& +\frac{\lambda}{16 \pi} \int d k_{1} d k_{2} d k_{3} d k_{4} \frac{\delta\left(k_{1}+k_{2}+k_{3}+k_{4}\right)}{\left(k_{1}+k_{2}\right)^{2}} \\
& \times\left(-2 f_{+}\left(k_{1}, k_{2}\right) f_{+}\left(k_{3}, k_{4}\right) M^{\dagger}\left(k_{1}, k_{4}\right) M\left(-k_{2},-k_{3}\right)\right. \\
& +f_{-}\left(k_{1}, k_{2}\right) f_{-}\left(k_{3}, k_{4}\right) M^{\dagger}\left(k_{1}, k_{4}\right) M^{\dagger}\left(k_{3}, k_{2}\right) \\
& \left.+f_{-}\left(k_{1}, k_{2}\right) f_{-}\left(k_{3}, k_{4}\right) M\left(k_{1}, k_{4}\right) M\left(k_{3}, k_{2}\right)\right) .
\end{aligned}
$$

\section{B. Renormalization}

The integral in (10) and subsequently the Hamiltonian contain a divergence and require regularization. For that, we regularize $\frac{1}{\left(k+k_{1}\right)^{2}}$ using the standard principal value (PV) prescription $\int d x \frac{f(x)}{(x-y)^{2}} \rightarrow \mathrm{PV} \int d x \frac{f(x)-f(y)}{(x-y)^{2}}+\frac{2 f(y)}{\epsilon}$.

It is readily seen that $\Pi^{-}$is finite but $\Pi^{+}$diverges as

$$
\Pi^{+}=\Pi_{r}^{+}+\frac{\lambda}{2 \pi \epsilon},
$$

with $\Pi_{r}$ finite. We have checked that, for physical states (on mass shell), the $\epsilon$ contributions cancel out (see below).

The solution to (10) that asymptotes $E_{k} \rightarrow|k|$ still suffers from a logarithmic divergence even after the PV prescription, namely,

$$
\frac{\lambda}{8 \pi E_{k}} \int d k_{1} \frac{E_{k_{1}}}{k_{1}^{2}}
$$

This is actually related to the mass divergence for the scalar one-loop self energy and renormalizes the scalar mass

$$
m_{r}^{2}=m^{2}+\frac{\lambda}{4 \pi} \int d k_{1} \frac{E_{k_{1}}}{k_{1}^{2}}
$$

From here on, we will refer to $\Pi^{+}$as the renormalized momentum operator, and $m$ as the renormalized mass, and omit the r-label for convenience. With this in mind, the renormalized integral equation (10) now reads

$$
\begin{aligned}
& \frac{k^{2}+m^{2}}{E_{k}}-E_{k} \\
& +\frac{\lambda}{4 \pi} \int d k_{1}\left(\left(\frac{E_{k_{1}}}{E_{k}}-\frac{E_{k}}{E_{k_{1}}}\right) \frac{\mathrm{PV}}{\left|k+k_{1}\right|^{2}}-\frac{E_{k_{1}}}{E_{k}} \frac{1}{k_{1}^{2}}\right)=0 .
\end{aligned}
$$

\section{WAVE-FUNCTION AND LIGHT-CONE LIMIT}

To construct the light-cone wave function of the scalar quarks, it is useful to recast the leading-order Hamiltonian in (12) in the form

$$
\begin{aligned}
H \approx & \int d p d q\left(\Pi^{+}(p)+\Pi^{+}(q)\right) M^{\dagger}(p, q) M(p, q) \\
& -\frac{\lambda}{16 \pi} \int d P \int d k d p \frac{A+B}{(p-k)^{2}},
\end{aligned}
$$

using the compact notation

$A=2 S_{+}(p, k, P) M^{\dagger}(p-P, p) M(k-P, k)$

$B=S_{-}(p, k, P)\left(M^{\dagger}(p, p-P) M^{\dagger}(k-P, k)+\right.$ c.c $)$

$S_{ \pm}(p, k, P)=f_{ \pm}(p-P, k-P) f_{ \pm}(p, k)$.

The bilocal mesonic operator $M(p, q)$ can be decomposed in suitably normalized modes 


$$
\begin{aligned}
M(p-P, p)= & \frac{1}{\sqrt{|P|}} \sum_{n}\left(m_{n}(P) \phi_{n}^{+}(p, P)\right. \\
& \left.-m_{n}^{\dagger}(-P) \phi_{n}^{-}(p-P,-P)\right),
\end{aligned}
$$

The first contribution refers to the light-cone wave function describing a pair of scalar quarks moving forward in the light front, while the second contribution refers to a pair moving backward in the light front. The pair is characterized by a relative momentum $p$ and a center of mass momentum $P$.

Here $m_{n}, m_{n}^{\dagger}$ are canonical bosonic annihilation and creation operators that satisfy the standard commutation rules, e.g.,

$$
\left[m_{n}(P), m_{l}^{\dagger}\left(P^{\prime}\right)\right]=\delta_{n l} \delta\left(P-P^{\prime}\right),
$$

and when applied to the ground state creates a meson state

$$
|P\rangle \equiv\left|E_{n}(P), P\right\rangle=\sqrt{2 E_{n}(P)} m_{n}^{\dagger}(P)|0\rangle,
$$

on mass shell $E_{n}(P)=\left(P^{2}+M_{n}^{2}\right)^{\frac{1}{2}}$. Equation (21) is seen to follow from the leading $1 / N_{c}$ commutation rules (6) provided that the wave functions $\phi_{n}^{ \pm}$satisfy pertinent ortonormality conditions, e.g.,

$$
\int d p\left(\phi_{n}^{+}(p, P) \phi_{l}^{+}(p, P)-\phi_{n}^{-}(p, P) \phi_{l}^{-}(p, P)\right)=\delta_{n l}|P| .
$$

The Heisenberg equation of motion follows by commutation

$$
i \partial_{t} M^{\dagger}(p-P, p)|0\rangle=\left[M^{\dagger}(p-P, p), H\right]|0\rangle,
$$

which mode-by-mode translates to

$$
\begin{aligned}
& \left(\Pi^{+}(p)+\Pi^{+}(P-p) \mp P_{n}^{0}\right) \phi_{n}^{ \pm}(p, P) \\
& =\frac{\lambda}{8 \pi} \int \frac{d k}{(p-k)^{2}} \\
& \quad \times\left(S_{+}(p, k, P) \phi_{n}^{ \pm}(k, P)-S_{-}(p, k, P) \phi_{n}^{\mp}(k, P)\right) .
\end{aligned}
$$

We checked that the $\epsilon$-dependent divergences noted in the momentum operator cancel out. Indeed, using (14) the LHS in (25) produces $\frac{\lambda}{\pi \epsilon} \phi^{ \pm}$, while the RHS in (25) produces $\frac{\lambda}{4 \pi \epsilon} S^{+}(k, k) \phi^{ \pm}=\frac{\lambda}{\pi \epsilon} \phi^{ \pm}$, both of which cancel out. This checks the consistency of the renormalization procedure for scalar QCD. No such renormalization is needed for spinor QCD.

In the large momentum limit $P$, the equation simplifies. For that we set $p=x P, k=y P$, and take $P \rightarrow \infty$ on both sides of (25). In this limit, the backward wave function vanishes $\phi_{-} \rightarrow 0$. Since

$$
\begin{aligned}
\Pi^{+} & (P x)+\Pi^{+}((1-x) P)-\sqrt{P^{2}+M_{n}^{2}} \\
& =\frac{1}{2 P}\left(\frac{m^{2}}{x}+\frac{m^{2}}{1-x}-M_{n}^{2}\right)+\mathcal{O}\left(\frac{1}{P^{2}}\right),
\end{aligned}
$$

and

$$
S_{+}(x P, y P, P)=\frac{(2-x-y)(x+y)}{\sqrt{x(1-x) y(1-y)}},
$$

the equation of motion (25) involves only the forward wave function in the form

$$
\begin{aligned}
& \left(\frac{m^{2}}{x}+\frac{m^{2}}{1-x}-M_{n}^{2}\right) \phi_{n}(x) \\
& \quad=\frac{\lambda}{4 \pi} \mathrm{PV} \int \frac{d y}{(x-y)^{2}} \frac{(2-x-y)(x+y)}{\sqrt{x(1-x) y(1-y)}} \phi_{n}(y),
\end{aligned}
$$

where we have defined $\phi_{n}^{+}(x P, P)=\phi_{n}(x)$, and PV refers to the principal value of the integral. (28) was obtained initially in the light-cone gauge in [5] using different arguments.

\section{QUASI-PARTON DISTRIBUTION FUNCTION}

The light-cone distribution for scalar quarks is just $\left|\phi_{n}^{+}(x)\right|^{2}$ in leading order in $1 / N_{c}$. We now show that to the same order, the light-cone distribution function and the quasidistribution function as defined in [1] are in agreement without further normalization. For that, we define the quasidistribution function

$$
\begin{aligned}
\tilde{q}(x, P)= & +i \int \frac{d z}{4 \pi} e^{i P x z}\left\langle P\left|\left(\partial_{1} \phi(z)\right)^{\dagger} W[z, 0] \phi(0)\right| P\right\rangle \\
& -i \int \frac{d z}{4 \pi} e^{i P x z}\left\langle P\left|(\phi(z))^{\dagger} W[z, 0] \partial_{1} \phi(0)\right| P\right\rangle,
\end{aligned}
$$

where $|P\rangle$ refers to the meson state. In the axial gauge, the Wilson line $W[z, 0]=1$. Using the mode decomposition (4) and the relations (11) we obtain for the quasidistribution

$$
\begin{aligned}
\tilde{q}(x, P)= & \frac{E_{n}(P)}{P} \frac{x P}{E(x P)} \\
& \times\left(\left|\phi_{n}^{+}(x P, P)\right|^{2}+\left|\phi_{n}^{+}(-x P, P)\right|^{2}\right. \\
& +\mid \phi_{n}^{-}\left(\left.(x P, P)\right|^{2}+\left|\phi_{n}^{-}(-x P, P)\right|^{2}\right) .
\end{aligned}
$$

For $P \rightarrow \infty$, we have $E_{n}(P) \rightarrow P$ and $E(x P) \rightarrow x P$ and all $\phi_{-}$vanish. The quasiparton distribution function reduces identically to the parton distribution function $\left|\phi_{n}^{+}(x)\right|^{2}$.

For finite $P$, (30) shows that the backward moving pair in $\phi^{-}$contributes. To assess this quantitatively, we now expand in $\frac{1}{P}$ the contributions $\phi^{ \pm}$in (30). For that, we go back to (25) and expand in $\frac{1}{P}$, namely 


$$
\begin{aligned}
\Pi^{+} & =|P|+\frac{m^{2}}{2|P|}+\frac{\beta_{1}}{2|P|^{3}}+\mathcal{O}\left(\frac{1}{|P|^{4}}\right) \\
E(P) & =|P|+\frac{\beta_{2}}{|P|}+\mathcal{O}\left(\frac{1}{|P|^{2}}\right) .
\end{aligned}
$$

The coefficients $\beta_{1}$ is fixed through a straightforward Taylor expansion of $\Pi^{+}$, while $\beta_{2}$ is fixed by the integral equation (10). Their explicit form is not needed for the general arguments to follow. With this in mind, the leading correction to $\phi^{-}$is

$\phi_{1}^{-}=P^{2} \phi_{n}^{-}(x)=\frac{\lambda}{24 \pi \sqrt{x(1-x)}} \int_{0}^{1} d y \frac{\phi_{n}(y)}{\sqrt{y(1-y)}}$,

and the subleading correction for $\phi^{+}=\phi(x)+\frac{1}{P^{2}} \phi_{1}^{+}(x)$ formally solves

$$
\tilde{\phi} \equiv\left(K_{0}-H_{0}\right) \phi_{1}^{+}=-K_{1} \phi+H_{1} \phi-H_{0}^{-} \phi_{1}^{-} .
$$

Here we have defined

$$
\begin{aligned}
K_{0}(x)= & \frac{m^{2}}{x}+\frac{m^{2}}{\bar{x}}-M_{n}^{2} \\
H_{0}(x, y)= & \frac{\lambda}{4 \pi} \frac{(\bar{x}+\bar{y})(x+y)}{\sqrt{x y \bar{x} \bar{y}}} \frac{1}{(x-y)^{2}} \\
K_{1}(x)= & \frac{\beta_{1}}{x^{3}}+\frac{\beta_{1}}{\bar{x}^{3}} \\
H_{0}^{-}(x, y)= & -\frac{\lambda}{4 \pi \sqrt{x y \bar{x} \bar{y}}} \\
H_{1}(x, y)= & \frac{1}{(x-y)^{2} \sqrt{x \bar{x} y \bar{y}}}+\beta_{2}\left(x^{2}-y^{2}\right)\left(\frac{1}{\bar{y}^{2}}-\frac{1}{\bar{x}^{2}}\right) \\
& +\beta_{2}\left(\bar{x}^{2}-\bar{y}^{2}\right)\left(\frac{1}{y^{2}}-\frac{1}{x^{2}}\right),
\end{aligned}
$$

with $\bar{x}=1-x$ and $\bar{y}=1-y$. In general, this equation is solved in the same Hilbert space that defines $K_{0}-H_{0}$, if we note that $K_{0}-H_{0}$ is hermitian in the space defined with the measure $\int \phi^{\dagger} \phi$ where the set of $\phi_{n}$ forms a complete basis set. The formal solution to (34) is

$$
\phi_{1}^{+}(x)=\sum_{m \neq n} \frac{\phi_{m}(x) \int_{0}^{1} d y \phi_{m}^{\dagger}(y) \tilde{\phi}(y)}{M_{m}^{2}-M_{n}^{2}} .
$$

The $\frac{1}{P}$ expansion now clearly shows that the rate at which the quasidistribution (30) approaches the asymptotic lightcone distribution $\left|\phi_{n}(x)\right|^{2}$ is smooth for all $x \neq 0,1$. It is singular for $x=0,1$ through the contribution of the backward moving pair $\phi^{-}$in (32). So the large $P$ limit should be taken before the $x \rightarrow 0,1$ limits at the edges.

\section{ALGEBRAIC STRUCTURE}

The algebraic framework (6) allows us to go beyond the leading order in $1 / N_{c}$, and therefore check the proposal in [1] beyond the leading order we have so far established. A solution to the algebraically closed set (6) can be found by organizing the bilocal operator in $1 / N_{c}$,

$$
\begin{gathered}
M=M^{0}+\frac{1}{N_{c}} M^{1}+\mathcal{O}\left(\frac{1}{N_{c}^{2}}\right) \\
N=N^{0}+\frac{1}{N_{c}} N^{1}+\mathcal{O}\left(\frac{1}{N_{c}^{2}}\right),
\end{gathered}
$$

where $M^{0}$ satisfies the commutation relation

$$
\left[M^{0}\left(k_{1}, k_{2}\right), M^{0 \dagger}\left(k_{3}, k_{4}\right)\right]=\delta\left(k_{1}-k_{3}\right) \delta\left(k_{2}-k_{4}\right),
$$

in the large $N_{c}$ limit. In terms of (36)-(37), the solution to (6) can be found by inspection in leading and next to leading order

$$
\begin{aligned}
N_{12}^{0} & =\int d 3 M_{13}^{0 \dagger} M_{23}^{0} \\
\bar{N}_{12}^{0} & =\int d 3 M_{31}^{0 \dagger} M_{32}^{0} \\
M_{12}^{1} & =\mp \frac{1}{2} \int d 3 d 4 M_{34}^{0 \dagger} M_{14}^{0} M_{32}^{0} \\
N^{1} & =0 .
\end{aligned}
$$

Here, we are using the short-hand notations $d 3,4 \equiv d k_{3,4}$, $M_{13} \equiv M\left(k_{1}, k_{3}\right)$ and so on. It is important to note that the expansion of the $N$ 's starts at second order. From now on to avoid cluttering, we omit the 0 for the large $N_{c}$ asymptotic operator.

When the operators in (38) are inserted back into the Hamiltonian, we obtain a complete expression for the first three terms of the $1 / N_{c}$ expanded Hamiltonian in terms of the large $N_{c}$ asymptotic operators that define the Hilbert space. Specifically and to order $\frac{1}{N_{c}^{2}}$, the various contributions to the Hamiltonian can be schematically written as

$$
\begin{aligned}
H \approx & K_{M M}^{00} M^{\dagger} M \\
& +\frac{1}{N_{c}} K_{M M}^{01}\left(M^{\dagger 1} M+M^{\dagger} M^{1}\right)+\frac{1}{N_{c}^{2}} K_{M M^{11}}^{11} M^{1 \dagger} M^{1} \\
& +\frac{K_{N M}^{00}}{\sqrt{N_{c}}} N M+\frac{K_{N M}^{01}}{N_{c} \sqrt{N_{c}}} N M^{1}+\frac{K_{N N}^{00}}{N_{c}} N N,
\end{aligned}
$$

with the $K^{\prime}$ s referring to pertinent coefficients (integrals of the wave functions). Thus, up to order $1 / N_{c}^{2}$ we encounter six $M$ interactions, but up to oder $1 / N_{c} \sqrt{N_{c}}$ we are still dealing with more tractable quartic and qubic terms. Our algebraic treatment differs notably from the one presented in [11] in that in ours the algebra is corrected which is 
required for a consistent expansion. The resulting effective hadronic Hamiltonian is different.

\section{CORRECTION TO THE PDF IN SPINOR QCD}

Thus far, our discussion has concentrated on twodimensional scalar QCD, where we have established that the quasiparton distribution function reduces to the parton distribution function in leading order in $1 / N_{c}$. We have checked that this is also the case for two-dimensional spinor QCD, in agreement with a recent study [8]. In the Appendix, we have briefly summarized the key changes from scalar to spinor in the light-cone and axial gauge.

Since in the spinor version, the underlying fields are fermionic and not bosonic, the algebraic structure (6) differs from scalar to spinor QCD only in the sign switch $s=+1 \rightarrow-1$, with exactly the same bosonized Hamiltonian (39). Also, to avoid unecessary long formula we will only discuss the $1 / N_{c}$ corrections to the parton distribution function in two-dimensional spinor instead of scalar QCD. The arguments for both models are similar, but the formula for scalar QCD are laboriously long as we have checked, with exactly the same conclusion.

Using the definitions for spinor QCD in the Appendix, the pertinent bilocal mesonic operator $M$ in the light-cone gauge takes now the form

$$
M(x P,(1-x) P)=\frac{1}{\sqrt{|P|}} \sum_{n} m_{n}(P) \phi_{n}(x)
$$

which satisfies (6) with $s=-1$. To order $1 / N_{c}$, the Hamiltonian for two-dimensional spinor QCD is the same as in (39), which after inserting (40) yields the first two leading contributions to the interaction of the form

$$
\begin{aligned}
& \frac{\lambda}{4 \pi \sqrt{N_{c}}} \int \frac{d P d P_{1}}{P^{\frac{3}{2}}} \\
& \quad \times\left(m_{i}^{\dagger}\left(P_{1}\right) m_{j}^{\dagger}\left(P-P_{1}\right) m_{k}(P) f_{i j k}\left(\frac{P_{1}}{P}\right)+\text { c.c }\right) \\
& \quad+\frac{1}{N_{c}} m^{\dagger} m^{\dagger} m m .
\end{aligned}
$$

The quartic contribution in (41) is only shown schematically. It is of order $1 / N_{c}$, and apparently relevant for the $1 / N_{c}$ correction to the parton distribution function. However, by simple inspection it gives zero contribution when acting on a free and leading meson contribution to the state, i.e.,

$$
\left(\frac{1}{N_{c}} m^{\dagger} m^{\dagger} m m\right) m^{\dagger}|0\rangle=0 .
$$

It will be dropped. Therefore, the leading correction to the parton distribution function is given by

$$
\begin{aligned}
& \sum_{k l} \int \frac{d k d q}{2 \pi} \phi_{k}\left(\frac{x P}{x P+q}\right) \phi_{l}\left(\frac{k}{k+q}\right) \\
& \quad \times^{1}\left\langle P_{i}\left|\left(\frac{m_{k}^{\dagger}(x P+q) m_{l}(k+q)}{\sqrt{(x P+q)(k+q)}}\right)\right| P_{i}\right\rangle^{1} .
\end{aligned}
$$

Here, $|P\rangle^{1}$ is the first-order perturbation of the meson state $m_{i}^{\dagger}(P)|0\rangle$, which by standard perturbation theory reads

$$
\begin{aligned}
|P\rangle^{1}= & \frac{\lambda}{2 \sqrt{2 \pi N_{c}}} \int d P_{1} \\
& \times \sum_{k l}\left(\frac{f_{k l i}\left(\frac{P_{1}}{P}\right) m_{k}^{\dagger}\left(P_{1}\right) m_{l}^{\dagger}\left(P-P_{1}\right)}{\frac{m_{k}^{2}}{x}+\frac{m_{l}^{2}}{1-x}-m_{i}^{2}}\right)|0\rangle .
\end{aligned}
$$

Inserting (44) into (43) and carrying out the contractions yields

$$
\delta q_{i}(x)=\frac{\lambda^{2}}{\pi^{2} N_{c}} \int_{0}^{1-x} d y \sum_{k k^{\prime} l} \frac{F_{k l i}(x, y) F_{k^{\prime} l i}(x, y)}{x+y}
$$

as a correction to the leading parton distribution function $q_{i}(x)=\left|\phi_{i}^{+}(x)\right|^{2}$, with

$$
F_{k l i}(x, y)=\frac{f_{k l i}(x+y) \phi_{k}\left(\frac{x}{x+y}\right)}{\frac{m_{k}^{2}}{x+y}+\frac{m_{l}^{2}}{1-x-y}-m_{i}^{2}},
$$

and

$$
\begin{aligned}
\frac{f_{i j k}(x)}{\sqrt{x(1-x)}}= & \int d x_{1} d x_{2} \frac{\phi_{i}\left(x_{1}\right) \phi_{j}\left(x_{2}\right) \phi_{k}\left(x+x_{2}-x x_{2}\right)}{\left(x x_{1}+x x_{2}-x-x_{2}\right)^{2}} \\
& -\int d x_{1} d x_{2} \frac{\phi_{i}\left(x_{1}\right) \phi_{j}\left(x_{2}\right) \phi_{k}\left(x_{2}-x x_{2}\right)}{\left(x x_{1}+x x_{2}-x-x_{2}\right)^{2}} .
\end{aligned}
$$

\section{CORRECTION TO THE QUASI-PDF IN SPINOR QCD}

In this section, we derive the $1 / N_{c}$ correction to the quasiparton distribution function for two-dimensional spinor QCD and show that it is in agreement with the $1 / N_{c}$ correction to the parton distribution we just established in the large momentum limit. For that, we switch to the description of two-dimensional spinor QCD in the axial gauge using the changes in the Appendix.

In the axial gauge, the Hamiltonian is written in terms of $m_{n}(P)$ and $\phi_{ \pm}$. The structure of the Hamiltonian is still of the form (39). We now note that the contributions to the first order shift of the state $|P\rangle^{1}$ of the form $m^{\dagger} m^{\dagger} m^{\dagger}$ always carries $\phi^{-}$. In the large momentum limit, these terms drop out as we have shown earlier, so they will be ignored. 
The only surviving terms in the Hamiltonian at large momentum are also of the form $m^{\dagger} m^{\dagger} m+$ c.c.

With the above in mind and to be more specific, the parts of the Hamiltonian (39) that will contribute to the quasiparton distribution function in leading order in perturbation theory are of the form

$$
\begin{aligned}
& H_{1}=\frac{1}{\sqrt{N_{c}}} \sum_{123} f_{123} m_{1}^{\dagger} m_{2}^{\dagger} m_{3} \\
& H_{2}=\frac{1}{N_{c}} \sum_{1234} f_{1234} m_{1}^{\dagger} m_{2}^{\dagger} m_{3}^{\dagger} m_{4} .
\end{aligned}
$$

The ensuing shifts caused by (48) on the mesonic state to first order in $\frac{1}{N_{c}}$ are, respectively, of the form

$$
|i\rangle^{1}=\frac{1}{\sqrt{N_{c}}} \sum_{12}|12\rangle \alpha_{12 i} \quad|i\rangle^{2}=\frac{1}{N_{c}} \sum_{123}|123\rangle \alpha_{123 i},
$$

with

$$
\begin{aligned}
\alpha_{12 i}= & \frac{f_{12 i}}{E_{1}+E_{2}-E_{i}} \\
\alpha_{123 i}= & \frac{f_{123 i}}{E_{1}+E_{2}+E_{3}-E_{i}} \\
& +\sum_{4} \frac{f_{123} f_{34 i}}{\left(E_{1}+E_{2}+E_{3}-E_{i}\right)\left(E_{3}+E_{4}-E_{i}\right)},
\end{aligned}
$$

and the coefficients $f_{i j k}$ and $f_{i j k l}$ are

$$
\begin{aligned}
f_{i j k}\left(P_{1}, P_{2}, P_{3}\right)= & \frac{\lambda}{4 \pi} \int d k_{1} d k_{2} d k_{3} d k_{4} d q \delta\left(k_{1}+k_{2}+k_{3}-k_{4}\right) \delta\left(k_{1}+k_{2}-P_{1}\right) \delta\left(k_{3}+q-P_{2}\right) \delta\left(k_{4}+q-P_{3}\right) \\
& \times\left(\frac{\phi_{i}^{+}\left(k_{1}, P_{1}\right) \phi_{j}^{+}\left(k_{3}, P_{2}\right) \phi_{k}^{+}\left(k_{4}, P_{3}\right) S\left(k_{1}, k_{2}, k_{3}, k_{4}\right)}{\left(k_{1}-k_{4}\right)^{2}}-\frac{\phi_{i}^{+}\left(k_{1}, P_{1}\right) \phi_{j}^{+}\left(q, P_{2}\right) \phi_{k}^{+}\left(q, P_{3}\right) S\left(k_{2}, k_{1}, k_{3}, k_{4}\right)}{\left(k_{1}+k_{3}\right)^{2}}\right) \\
& +f_{i j k}^{-},
\end{aligned}
$$

where we have set

$S\left(k_{1}, k_{2}, k_{3}, k_{4}\right)=\cos \left(\frac{\theta\left(k_{1}\right)-\theta\left(k_{4}\right)}{2}\right) \sin \left(\frac{\theta\left(k_{2}\right)+\theta\left(k_{3}\right)}{2}\right)$.

The last contribution $f_{i j k}^{-}$involves at least one $\phi^{-}$and therefore drops out in the large momentum limit, so it will not be quoted.

All contributions of the form $f_{i j k l}$ involve at least one $\phi^{-}$ and also drop out in the large momentum limit. More specifically, in the large momentum limit, we set $P_{i}=$ $P \rightarrow+\infty$, and we change our variables to $P_{1}=x P$, $P_{2}=y P$, and $P_{3}=z P$, then any term which contains $\phi^{-}\left(x_{1} P, x_{2} P\right)$ vanishes in this limit, an example is the $f_{1234}$ term.

The parton fractions are constrained kinematically. For instance, the energy denominator

$$
\frac{1}{E_{x P}+E_{y P}+E_{z}-E_{p}}
$$

implies $0<x, y, z<1$ in leading order in $1 / P$, otherwise the contribution is subleading. In this case, the only term in $H^{1}$ which contains only $\phi^{+}$[first contribution in (39)] will reduce to the light-cone gauge term if one identifies the creation operators in both cases using

$$
\begin{aligned}
\phi_{n}^{+}(x P, P) & \rightarrow \phi_{n}(x) \\
\frac{1}{E_{x P}+E_{(1-x) P}-E_{P}} & \rightarrow \frac{2 P}{\frac{m_{1}^{2}}{x}+\frac{m_{2}^{2}}{1-x}-m_{i}^{2}} .
\end{aligned}
$$

More specifically, the first order correction to the quasiparton distribution function is proportional to

$$
\begin{aligned}
& \left\langle P\left|\int d p d q \sin \left[\frac{\theta(x P)+\theta(p)}{2}\right] M^{\dagger}(x P, q) M(p, q)\right| P\right\rangle \\
& \quad+\langle P| \int d p d q \sin \left[\frac{\theta(x P)+\theta(p)}{2}\right] \\
& \quad \times M^{\dagger}(q,-p) M(q,-x P)|P\rangle,
\end{aligned}
$$

with $|P\rangle$ corrected to first order. There are two type of contributions in (54) as we now discuss.

First, the $m^{\dagger} m$ term. For this only the $|i\rangle^{1}$ in the shift of the state contributes, and the specific contribution with only $\phi^{+}$is

$$
\begin{aligned}
& 2 \sin \frac{\theta(x P)}{2} \sum_{k k^{\prime} l} \frac{\alpha_{k l i} \alpha_{k^{\prime} l i}}{|k|} \phi_{k}^{+}\left(x P, p_{k}\right) \phi_{k^{\prime}}^{+}\left(x P, p_{k}\right) \\
& +2 \sin \frac{\theta(x P)}{2} \sum_{k k^{\prime} l} \frac{\alpha_{k l i} \alpha_{k^{\prime} l i}}{|k|} \\
& \quad \times \phi_{k}^{+}\left(x P+p_{k}, p_{k}\right) \phi_{k^{\prime}}^{+}\left(x P+p_{k}, p_{k}\right) .
\end{aligned}
$$

In the large momentum limit, we have $p_{k}=y P$, and $p_{l}=$ $(1-y) P$ as discussed above. The first term is nonzero if 
$0<x<y$, and the second term is always zero for $0<x<1$ since $(x+y)>y$. Thus by shifting $y \rightarrow y+x$ with $0<y<1-x$, and taking care of factors of $P$, this contribution matches the correction to the parton distribution function in the light-cone gauge (45).

Second, the $m m+m^{\dagger} m^{\dagger}$ term comes with at least one $\phi^{-}$, and is always zero in the large $P$ limit as discussed above. It follows, that the order $1 / N_{c}$ contribution to the quasiparton distribution matches the parton distribution in the large momentum limit without renormalization in twodimensional spinor QCD. We have explicitly checked that the same holds for two-dimensional scalar QCD.

\section{CONCLUSIONS}

Using a bosonized form of two-dimensional scalar and spinor QCD, we have analyzed the quasiparton distribution of a meson state. In the infinite momentum limit, the quasidistribution matches the parton distribution on the light cone both in leading and subleading order without further renormalization, but the limit is subtle at the parton fractions $x=0,1$. This provides a nonperturbative check on the proposal put forth by one of us [1] for extracting the QCD light-cone partonic distributions from their quasidistribution counterparts using pertinent equal-time Euclidean correlators through suitable matching at large momentum.

\section{ACKNOWLEDGMENTS}

This work was supported by the U.S. Department of Energy under Contract No. DE-FG02-93ER-40762 for X. J. and No. DE-FG-88ER40388 for Y. L. and I.Z.

\section{APPENDIX: TWO-DIMENSIONAL SPINOR QCD IN THE LIGHT-CONE AND AXIAL GAUGE}

Here and for convenience we briefly summarize some of the changes needed to recover spinor QCD from scalar
QCD as developed in the main text. Both in the light-cone and axial gauge, the mesonic operators $M$ and $N$ are defined as in Sec. II A with $s=-1$. The fermionic fields in terms of creation-annihilation operators are defined as

$\psi(x)=\int_{0}^{\infty} \frac{d p^{+}}{2 \pi}\left(a\left(p^{+}\right) e^{-i p^{+} x^{-}}+b^{\dagger}\left(p^{+}\right) e^{-i p^{+} x^{-}}\right)$
$\psi(x)=\int \frac{d p}{2 \pi} e^{i p x}\left(a(p) u(p)+b^{\dagger}(-p) v(-p)\right)$,

in the light-cone and axial gauge, respectively, with the two-dimensional spinors

$$
\begin{aligned}
u(p) & =e^{-\frac{1}{2} \theta(p) \gamma^{1}}(1,0)^{T} \\
v(-p) & =e^{-\frac{1}{2} \theta(p) \gamma^{1}}(0,1)^{T} .
\end{aligned}
$$

The angle $\theta(p)$ solves the transcendental equation [12]

$p \cos (\theta(p))-m \sin (\theta(p))=\frac{\lambda}{2} \mathrm{PV} \int d k \frac{\sin (\theta(p)-\theta(k))}{(p-k)^{2}}$.

The mode decomposition in the light-cone gauge is given in (40), and in the axial gauge it is

$$
\begin{aligned}
& M\left(k_{1}, P-k_{1}\right)=\frac{1}{\sqrt{|P|}} \\
& \quad \times \sum_{n}\left(\phi_{n}^{+}\left(k_{1}, P\right) m_{n}(P)-\phi_{n}^{-}\left(-k_{2},-P\right) m_{n}^{\dagger}(-P)\right) .
\end{aligned}
$$

The bosonized Hamiltonian is still of the form (39), with the relevant $M^{\dagger} M M$ term given in the main text.
[1] X. Ji, Phys. Rev. Lett. 110, 262002 (2013); Sci. China Phys. Mech. Astron. 57, 1407 (2014).

[2] Y. Hatta, X. Ji, and Y. Zhao, Phys. Rev. D 89, 085030 (2014); J. W. Chen, S. D. Cohen, X. Ji, H. W. Lin, and J. H. Zhang, Nucl. Phys. B911, 246 (2016); J. H. Zhang, J. W. Chen, X. Ji, L. Jin, and H. W. Lin, Phys. Rev. D 95, 094514 (2017).

[3] C. Alexandrou, K. Cichy, M. Constantinou, K. Jansen, A. Scapellato, and F. Steffens, Phys. Rev. Lett. 121, 112001 (2018).

[4] S. S. Shei and H. S. Tsao, Nucl. Phys. B141, 445 (1978).

[5] T. N. Tomaras, Nucl. Phys. B163, 79 (1980).
[6] B. Grinstein, R. Jora, and A. D. Polosa, Phys. Lett. B 671, 440 (2009).

[7] G. 't Hooft, Nucl. Phys. B75, 461 (1974).

[8] Y. Jia, S. Liang, X. Xiong, and R. Yu, Phys. Rev. D 98, 054011 (2018).

[9] P. Ring and P. Schuck, The Nuclear Many-Body Problem (Springer, New York, 1980).

[10] K. Itakura, Phys. Rev. D 54, 2853 (1996); Y. S. Kalashnikova and A. V. Nefediev, Usp. Fiz. Nauk 172, 377 (2002) [Phys. Usp. 45, 347 (2002)].

[11] J. L. F. Barbon and K. Demeterfi, Nucl. Phys. B434, 109 (1995).

[12] I. Bars and M. B. Green, Phys. Rev. D 17, 537 (1978). 